\title{
Estudiantes de primera generación y el impacto socioeconómico en sus familias, caso facultad de ciencias contables, universidad nacional de Pilar, años 2011 a 2021
}

\author{
Rossana Carrasco \\ rossanahcarrasco2@gmail.com \\ Facultad de Humanidades y Ciencias de la Educación, \\ Universidad Nacional de Pilar.
}

\section{RESUMEN}

El objetivo de esta investigación es analizar los efectos socioeconómicos que ha generado en las familias el primer graduado universitario específicamente de la Universidad Nacional de Pilar, dentro del seno familiar, en un contexto en que la oferta de Educación Superior ha aumentado y a su vez la hace más accesible. Dentro de la construcción conceptual de la investigación, se considera la reflexión sobre la vinculación de los egresados de primera generación (EPG) y el impacto socioeconómico que genera en el entorno. Se estudia el cumplimiento de los objetivos de formación y de la misión de la universidad, en donde el sistema educativo debe estar integrado a su vez al contexto sociocultural. El enfoque es cuantitativo, por cuanto que se analizan datos documentales y se aplicar una encuesta mediante un cuestionario autoadministrado, vía Google Forms basado en los Indicadores de Progreso Nacional Social implementado a mundial. La población la constituyen los Egresados de la Primera Generación, y la muestra es de tomada de manera intencional, considerando que solo los que responden a la condición de EPG, son incluidos en este trabajo. Finalmente, se discuten los resultados a partir de los cuales se contrasta la hipótesis. Con los resultados se comprueba que los EPG, han generado un impacto socioeconómico positivo en las familias.

Palabras clave: estudiante universitario de primera generación; formación; socioeconómico; movilidad social. 


\title{
First-generation students and the socio-economic impact of their families, case of the faculty of accounting sciences, universidad nacional de Pilar, years 2011 to 2021
}

\begin{abstract}
The objective of this research is to analyze the socioeconomic effects that the first university graduate, specifically from the National University of Pilar, has generated on families, within the family, in a context in which the offer of Higher Education has increased and in turn makes it more accessible. Within the conceptual construction of the research, the reflection on the bonding of first generation graduates (EPG) and the socioeconomic impact it generates on the environment is considered. The fulfillment of the training objectives and the mission of the university is studied, where the educational system must be integrated in turn to the sociocultural context. The approach is quantitative, since documentary data are analyzed and a survey is applied through a self-administered questionnaire, via Google Forms based on the National Social Progress Indicators implemented worldwide. The population is made up of First Generation Graduates, and the sample is taken intentionally, considering that only those who respond to the EPG condition are included in this work. Finally, the results from which the hypothesis is tested are discussed. The results show that the EPGs have generated a positive socioeconomic impact on families
\end{abstract}

Keywords: first generation university student; training; socioeconomic; social mobility

Artículo recibido: 15 octubre. 2021 Aceptado para publicación: 18 noviembre 2021 Correspondencia: rossanahcarrasco2@gmail.com Conflictos de Interés: Ninguna que declarar 


\section{INTRODUCCIÓN}

La Universidad es una institución, que, a más de aportar para el desarrollo de un país, con las funciones sustantivas de docencia, investigación y extensión, debe constituirse en una institución central generadora de inclusión y de ascenso social; eso solo puede ser posible implementando un modelo universitario inclusivo para el sistema de ingreso a la misma. Estos alumnos tienen que ser un objetivo prioritario de las instituciones de educación superior, ya que los mismos llegan muchas veces con menor "capital cultural" (Bourdieu, 1964) en comparación a compañeros que "provienen de familias con mayor nivel educativo y económico" (Arocena, 2014). Cuando se habla de capital cultural se hace referencia a instrumentos de conocimiento, a saber, hacer, a habilidades y técnicas que los jóvenes heredan de su medio familiar y que contribuyen con su éxito académico.

Las familias paraguayas, de un tiempo a esta parte, han visto a la educación superior como el camino para la movilidad social ascendente y, por tanto, se esfuerzan para que alguno de sus miembros pueda acceder a ella. Históricamente ha sido parte de la idiosincrasia, el hecho de que la manera de prosperar lo constituye la formación universitaria, garantizando un mejor futuro: mayores ingresos, más prestigio, más satisfacción, distinción. El gran impulsor de esta realidad parece ser la movilidad social. La movilidad social ha sido entendida tradicionalmente como la creencia que los tránsitos entre las categorías sociales son permeables, por lo que sería factible movilizarse hacia un grupo de estatus superior o inferior (Tajfel, 1981). Tal desplazamiento estaría, en la actualidad, asociado a la noción de igualdad de oportunidades.

La primera generación de los estudiantes posee otras características demográficas, tales como el bajo nivel socioeconómico y menor tasa de inscripción, que están asociados con la deserción. Todos estos factores y las interacciones entre ellos aumentan el riesgo de reprobar de los estudiantes de primera generación lo cual impide permanecer en la educación postsecundaria, con relación a otros pares de la generación continua (Choy2001; Lohfink y Paulsen 2005).

El objetivo de este trabajo es identificar en qué medida impacta en las familias el EPG, a la vez de caracterizar su nivel sociodemográfico, que servirá para la toma de 
decisiones en los ámbitos para plantear políticas de Estado, que coadyuven con este proceso.

La situación de los egresados da cuenta, junto con otros factores, de las bondades del programa, del cumplimiento de los objetivos de formación y de la misión de la universidad, en donde el sistema educativo en que se encuadra, debe estar integrado a su vez en un determinado contexto sociocultural con su específica filosofía política y educativa.

Por lo antes expuesto, el epicentro sobre el cual se basó el planteamiento de esta investigación, se fundamenta principalmente en la falta de datos cualitativos y cuantitativos en torno al impacto socioeconómico que genera la formación universitaria de los jóvenes, en la sociedad pilarense, más específicamente del primer graduado universitario, dentro del seno familiar.

Dentro de la construcción conceptual de la investigación se considera la reflexión sobre la vinculación de los egresados de primera generación de la Universidad Nacional de Pilar y el impacto socioeconómico que genera en el entorno en cual se desarrolla.

\section{REVISIÓN BIBLIOGRÁFICA}

\section{Antecedentes del tema}

Es sabido que el nivel educativo de una población está intrínsecamente ligado al nivel de desarrollo socioeconómico y político alcanzado por el país. Es así que, las consecuencias positivas generadas por el sistema educativo se integran y se asumen tanto a nivel personal como a nivel del país, de manera tal que una nación con un nivel de educación más desarrollado generará mejores relaciones sociales, y mayor productividad reflejada en el crecimiento económico. La actitud de la juventud con relación a su oportunidad laboral se genera a partir de las señales que recibe del mercado de trabajo en correlación a lo que podría obtener con la capacitación. Es decir, el esfuerzo económico empleado en alcanzar mayor educación, está en función al retorno esperado del mismo (DGEEC/GTZ, 2003).

Allen, (2003) señala que la función principal de la universidad consiste en preparar a la gente para desempeñar un papel en la sociedad, y más concretamente, en el mercado laboral. De esta manera, la inserción laboral se constituye en un aspecto que involucra tanto al sector productivo como a la universidad. 
Más recientemente en nuestro medio, en un estudio sobre la educación universitaria en el Paraguay, al concluir sobre la situación del empleo profesional y el ingreso a la universidad, se ha encontrado que en el país es cierto aún que quienes ocupan puestos de prestigio y obtienen buenos ingresos económicos, en una apreciable cantidad, son egresados universitarios: sin embargo, se observa cada vez más que el número de los graduados universitarios crece a un ritmo mucho más elevado que la capacidad real de absorción/expansión que poseen esos niveles de prestigio y de buena remuneración económica. Los profundos cambios que se hicieron evidentes en la última década llevaron a repensar el problema de las relaciones entre educación superior y empleo, que tradicionalmente se planteaba en términos de una "sobreoferta" consecuencia de la explosión de la matrícula desde los años 50, que no estuvo acompañada en igual medida por un aumento de los puestos de trabajo adecuados a esas calificaciones. Ahora los estudios ponen el acento en otras cuestiones, entre ellas el riesgo de un aumento general del desempleo como consecuencia del auge de las nuevas tecnologías. (OEI; 1993).

La enseñanza superior actúa como agente que facilita el acceso a mayores oportunidades laborales cuando proporciona una formación completa y adecuada a las necesidades del egresado. No obstante, los procesos de inserción laboral de los graduados universitarios se caracterizan por su alto nivel de heterogeneidad ya que existen multitud de factores académicos, laborales y personales que pueden repercutir en sus oportunidades.

Los padres esperan que, gracias a la educación, sus hijos consigan un mejor empleo: "menos fatigante, menos sucio, menos mal pagado y más valorizado que el suyo" (Lahire, 1995, p. 398). Charlot (1999) plantea que el oficio de los padres funciona como "una referencia (positiva o negativa) en la construcción del sujeto" (p. 259), pero que en sí mismo no es relevante, sino el nivel de vida que conlleva. Esto es clave para los estudiantes ya que su aspiración es tener una vida diferente a la de sus padres.

La fuerte asociación del nivel socio-económico entre padres e hijos se manifiesta en la compleja ruptura de la transmisión intergeneracional de la pobreza. Investigando este tema en Israel, Gofen (2009) obtuvo resultados que sugieren que la familia tiene un rol clave para los EPG, constituyéndose más bien en una fuente de recursos que de restricciones. De acuerdo a su análisis, el mecanismo para romper la transmisión de la pobreza se desarrolla en tres aspectos. Primero, los padres otorgan un papel fundamental 
a la educación de los hijos, inscrita en un "horizonte temporal" de larga duración. Enseguida, las "relaciones interpersonales" referida a la disposición de los padres a realizar "sacrificios" por sus hijos para favorecer el acceso a la universidad. Finalmente, "valores de la familia", donde destacan la solidaridad y el respeto a los padres. Estos tres elementos confluyen en la implicación de los padres para promover los estudios superiores de sus hijos.

\section{Estudiantes de Primera Generación (EPG).}

El concepto de estudiante de primera generación, desde ahora EPG para este trabajo, se aborda desde distintas perspectivas, es un concepto polisémico, porque se lo ha estudiado desde diversas perspectivas. Los EPG son definidos como aquellos estudiantes de estatus socioeconómico bajo cuyos padres o tutores no han asistido o no se graduaron de alguna universidad. (Bórquez, 2017)

Existen múltiples sentidos y significados que vivencian aquellos jóvenes que experimentan el acceso a las universidades, y más aún si constituye ser el primero en la familia, ya que son fundamentales para el desarrollo integral de su formación socioeconómica y cultural.

Implica, además, pensar en una reciprocidad entre Estado y sociedad civil para delinear políticas públicas que influyan de manera positiva y significativa en el conjunto de la población. Sus trayectorias, acompañadas de una fuerte presencia estatal, son instancias de disputas que hay que acompañar y profundizar para que el acceso a la educación superior sea apropiado plenamente, en todas sus configuraciones posibles, por toda la comunidad. Por ello, la defensa de la educación pública no arancelada es un gran desafío y un compromiso ético y político. /AC-FACSO, como se citó en (Universidad Nacional de Centro de la Provincia de Buenos Aires).

Para el año lectivo 2021, se ha logrado legalizar y reglamentar la Ley 6629/2020 de “arancel cero" para las universidades públicas del Paraguay. La Ley sancionada por el Congreso Nacional establece la gratuidad de los cursos de admisión y de grado en todas las universidades públicas del país, en el instituto superior de bellas artes, instituto nacional de educación superior, en institutos de formación docente, dependientes del ministerio de educación y ciencias y en el instituto nacional de salud, para los egresados de la Educación Media de instituciones educativas del sector oficial o instituciones educativas del sector subvencionado. A pesar que el Decreto $N^{\circ} 4734$ del Ministerio de 
Hacienda, no ha reglamentado conforme a la propuesta inicial, es un comienzo para propiciar el acceso a los jóvenes provenientes de sectores carenciados económicamente (Congreso Nacional del Paraguay, 2021).

De lo expresado, conlleva a pensar en qué medida las políticas relacionadas con la gestión universitaria, convergen con las del Estado para esperar que el estudiante que ha adquirido una inserción con arancel cero, prevé las condiciones para que éstos puedan permanecer en el Sistema de la Educación Superior.

El presente trabajo aborda a los EPG como aquellos estudiantes que llegan a ser los primeros en su familia en asistir a la universidad y los desafíos y limitaciones con los que se encuentran y constituyen verdaderas barreras para la culminación de sus estudios.

Los EPG has sido objeto de estudios por sus características y los factores que influyen en el tiempo que estos causar alguna carrera de educación superior. (Ishitani, 2003) define a los EPG como estudiantes cuyos padres o encargados no han logrado egresar de una institución de educación superior, estos estudiantes particularmente proceden de hogares de ingresos bajos según (Flanagan, 2016) y, para (Choy, 2001) por más que sean implementadas políticas para promover una mayor equidad en términos de educación educativa, ellos permanecen en un sector social y económico desventajado.

\section{Avance de la educación superior.}

El fenómeno de la expansión de la educación superior ha sido impulsado por una fuerte demanda del acceso, debido al incremento de los retornos positivos que esto conlleva, sobre todo en los países con bajos niveles de escolaridad donde solo una pequeña proporción de la población logra acceder al sistema educacional, lo que hace que estudiantes y familias estén dispuestos a asumir una carga financiera esperando el retorno de su inversión. Asimismo, la expansión de la educación superior privada ha creado oportunidades para un nuevo grupo social caracterizado por provenir de familias de bajos ingresos y de menor capital cultural, lo cual conlleva a que muchos estudiantes de familias con baja calificación académica se matriculen en instituciones privadas, debido a la dificultad para acceder a las más prestigiosas universidades públicas (Teixeira, Landoni, 2017). El surgimiento de instituciones privadas de educación superior, por ende, está vinculado al interés por masificar el ingreso a la educación superior (Zhen, Domínguez, 2017) respondiendo a esta demanda de acceso. 
La expansión de las clases medias urbanas es clave para comprender el rápido crecimiento de las tasas de participación en la educación superior en muchos países durante los últimos 15 años. La relación entre la demanda social y la oferta de educación superior se encuentra mediada a su vez por el crecimiento y diversificación de las economías, decisiones del Estado, e incluso inversiones estatales, por instituciones educativas y certificaciones en expansión (Marginson, 2017). En esta línea, (Trow, 1973) planteó que el propósito de la educación superior pasó de ser exclusivamente de élite, donde se les formaba la mente y el carácter a las clases dominantes, a ser de masas, con el fin preparar a grandes grupos en habilidades técnicas y profesionales, para adaptar a la población en su conjunto a los cambios sociales y tecnológicos propios de una sociedad industrial avanzada, llegando a ser considerada como una obligación para las familias de las clases medias. (Araneda, et. al., 2018)

Sin embargo, algunas instituciones de educación superior y sus respectivos programas se diferencian cada vez más manteniendo la selectividad y el prestigio, mientras que otras de menor prestigio son las que reciben un alto número de estudiantes provenientes de familias de clases trabajadoras, que pertenecen a la primera generación de sus familias que acceden a la educación superior. Por lo tanto, esta mayor participación que es percibida como un resultado positivo, oculta una creciente diferenciación en los tipos de programas e instituciones, provocando una progresiva estratificación en el sistema (Courtois, 2018). De este modo, por ejemplo, en Alemania las instituciones universitarias que han expandido su matrícula recientemente se identifican de manera uniforme por representar la más alta educación y mantener así el carácter elitista de su expansión (Stock, 2018). En América Latina, el sector privado abastece a la mayor parte de los estudiantes, existiendo varios ejemplos de contribución positiva para el acceso. La diferencia entre las universidades públicas y privadas es, frecuentemente, más elocuente cuando se comparan los niveles de escolaridad de los padres, ya que estas últimas instituciones reciben a una mayor proporción de estudiantes cuyos padres tienen menor nivel de escolaridad (Teixeira, Landoni, 2017). Según (O’Shea, 2016), los alumnos que forman parte de la primera generación que accede a la universidad, están en desventaja en términos de preparación, tienen más problemas para transitar desde la educación secundaria a la universidad y presentan mayores problemas de deserción; a su vez, tienen deficientes resultados académicos. Sin embargo, es importante consignar 
que las desigualdades estructurales, en el caso de los estudiantes de primera generación, no sean enmascaradas como déficits individuales (Araneda, et. al., 2018).

(Carnoy, 2017) precisa que la expansión de la educación superior en Brasil, Rusia, India y China no ha implicado una igual distribución en los ingresos; es más, sin políticas de gasto fiscal y social orientadas a la reducción de la desigualdad de los ingresos, ésta continuará siendo alta e incluso puede llegar a aumentar.

En Chile, la creciente expansión de la matrícula a partir del año 1990 implicó un mayor acceso para los estudiantes provenientes de los distintos grupos socioeconómicos, abarcando tanto los grupos de mayores como de menores ingresos, junto con la continuidad de los niveles de estratificación social en este fenómeno, manteniéndose, por tanto, los niveles de desigualdad (Brunner, 2015). Asimismo, la proporción de educación superior privada se incrementó de manera importante, atendiendo este tipo de instituciones a los dos tercios de la población estudiantil de todos los tipos de quintiles de ingreso, mientras que las universidades pertenecientes al Consejo de Rectores de Universidades Chilenas (CRUCH) solo logran matricular alrededor de un tercio de dicha población, debido posiblemente a la creación del mecanismo de financiamiento denominado 'Crédito con Aval del Estado', que expandió la opción de optar al crédito para financiar los estudios universitarios a los estudiantes de universidades privadas a partir del año 2006 (Espinoza, González, 2015). En efecto, Estado e instituciones de educación superior son quienes estructuran conjuntamente el sistema de educación superior, estableciendo las formas de provisión y su costo y valor. Por lo tanto, el rol en la asignación social varía de sistema en sistema, determinando hasta qué punto la educación superior hace que la sociedad sea más igual o si reproduce las desigualdades existentes (Marginson, 2016).

En América Latina, (Espinoza, González, 2017) afirman que el aumento en las matrículas de los estudiantes durante las últimas treinta años no ha logrado equidad en el acceso, pues en las tasas de participación persisten las diferencias entre los grupos de ingresos y los estudiantes desventajados en términos de género u origen étnico, quienes ingresan a instituciones de educación superior de menor prestigio, generalmente orientadas a la capacitación técnico profesional (Araneda, et. al., 2018). 


\section{METODOLOGÍA}

\section{Tipo de investigación}

La modalidad de la investigación es: Documental y de Campo; los niveles son: descriptivo y explicativo y las fuentes utilizadas: primaria y secundaria.

El tipo de investigación, según el conocimiento que se desea alcanzar: descriptiva y explicativa, mientras que según la naturaleza de la información: cuantitativa.

La investigación se inicia con la Investigación Bibliográfica/ Documental: a través del método de lectura y revisión de textos y publicaciones, obtenidos de sitios de Bibliotecas Virtuales, además de consultas en los repositorios avezados sobre el tema. Se complementan con informaciones secundarias solicitadas por la Dirección General Académica del Rectorado de la Universidad Nacional de Pilar, la misma fueron trabajada en función de los objetivos perseguidos por el estudio.

Posteriormente se procedió al trabajo de campo, con la aplicación de encuestas a los EPG; la misma fue completada por los mismos en el lugar donde se desempeñan a fin de conocer las características de los egresados en torno al ámbito donde se desarrollan. Y la respectiva entrevista a las familias para conocer el impacto generado del EPG.

\section{Población y muestra.}

\subsection{Población}

La población está compuesta por egresados que sean considerados egresados de primera generación entre aquellos graduados entre los años 2011 y 2021 de la Facultad de Ciencias Contables, Administrativas y Económicas de la Universidad Nacional de Pilar.

\subsection{Muestra}

La muestra fue seccionada según un método de muestreo probabilístico, a modo de lograr la generalización de los resultados a la población de egresados, dicho modelo consta del siguiente procedimiento: 
Figura $\mathbf{N}^{\circ}$ 1: Procedimiento de selección de la muestra.

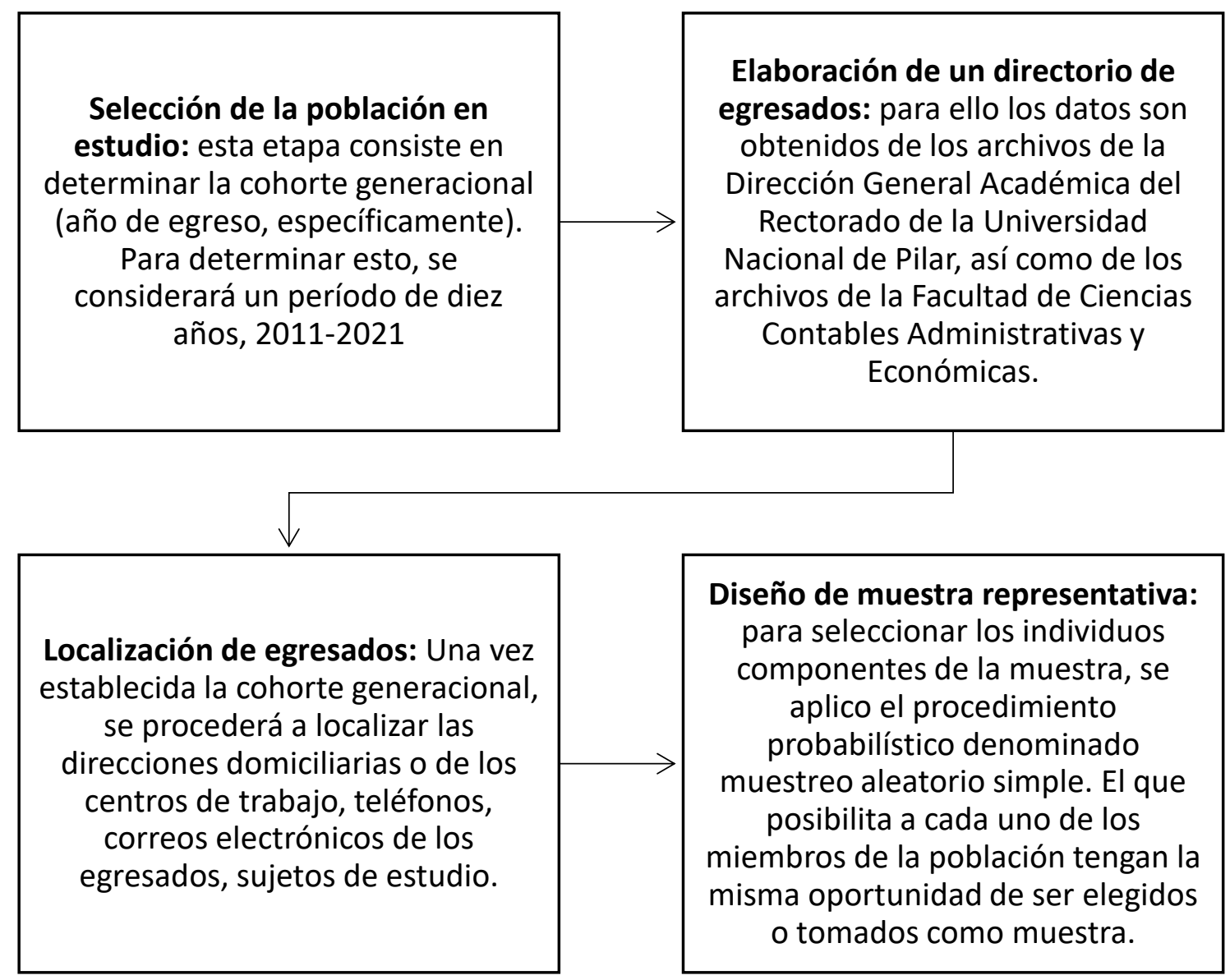

Fuente: Elaboración Propia.

\section{Métodos, técnicas e instrumentos de recolección de la información.}

Los métodos utilizados fueron: el Inductivo, la Síntesis, y el método Analítico; mientras que la técnica a ser utilizada será la encuesta. (Hernández y otros, 2014)

Se utilizaron como fuente primaria la "encuesta" en base a la aplicación del instrumento de recolección de datos que es el "cuestionario"- cuestionarios semiestructurado predominantemente con preguntas cerradas.

La aplicación del mismo se realizó mediante un cuestionario de Google Forms, enviado a través de correo electrónico y/o por correo electrónico, previa ubicación de los mismos a través de una planilla de elaboración propia.

\section{Procesamiento de los datos - Procedimientos.}

Una vez aplicado el cuestionario, se procedió a recopilar las informaciones, a través de la agrupación, organización y clasificación de las mismas, mediante un soporte estadístico que permite su sistematización y tabulación conforme a los registros 
obtenidos mediante el formulario, lo cual quedo presto mediante las tablas que sirvieron para una eficiente interpretación posterior.

\section{Análisis e interpretación de la información}

El estudio se centró en la recolección de datos primarios, ya que se originaron con el propósito específico de atender el problema que dio origen a la investigación de referencia.

Una vez tabulados los datos y representados en tablas y figuras, se procedió al análisis e interpretación de los mismos, utilizando estadísticos que faciliten su interpretación y relación. Se utilizo una tabla de contingencia relacionando la variable dependiente con las independientes y sus factores asociados, para contrastarlos con la hipótesis planteada en este trabajo.

\section{RESULTADOS Y DISCUSIÓN}

Se presentan los resultados obtenidos a través de los instrumentos de recolección de datos y el respectivo análisis de los mismos.

En la Fig. $\mathrm{N}^{\circ} 1$ se puede apreciar que los padres de egresados han realizado sus estudios universitarios en un 33\%, las madres en un 52\% mientras que sus hermanos en un $72 \%$, lo que indica que los hijos o hermanos menores de quienes hayan cursado sus estudios universitarios han tenido mejor oportunidad de alcanzar dicha formación.

Es importante diferenciar entre un EPG y uno que sea de segunda generación como mínimos, es decir; que uno de sus padres o miembro de su familia haya cursado sus estudios universitarios.

De acuerdo con (Chen, 2005), los EPG son alumnos menos propensos a matricularse en alguna institución postsecundaria que aquellos con padres o tutores con educación universitaria, los llamados estudiantes de segunda generación. Por lo general, los educandos de segunda generación tienden a asistir de inmediato a una institución de educación superior después de graduarse de la escuela secundaria y completar su licenciatura en el tiempo estimado, es decir, en cuatro o cinco años y a la edad de 22 o 23 años; mientras cursan, procuran no tener hijos, permanecen dependientes económicamente de otros y consideran los estudios como su principal responsabilidad, lo cual les permite dedicarse a su condición de escolares.

(Terenzini et al., 1996) afirman que en contraste, los EPG necesitan más tiempo para completar los objetivos académicos de una carrera o poseen menos probabilidades de 
graduarse debido a la falta de apoyo familiar, a las tensiones económicas, a la mala preparación académica y a otras barreras socioeconómicas.

Figura $\mathbf{N}^{\circ}$ 1: Nivel de estudios de padre, madre y hermanos de egresado/a.

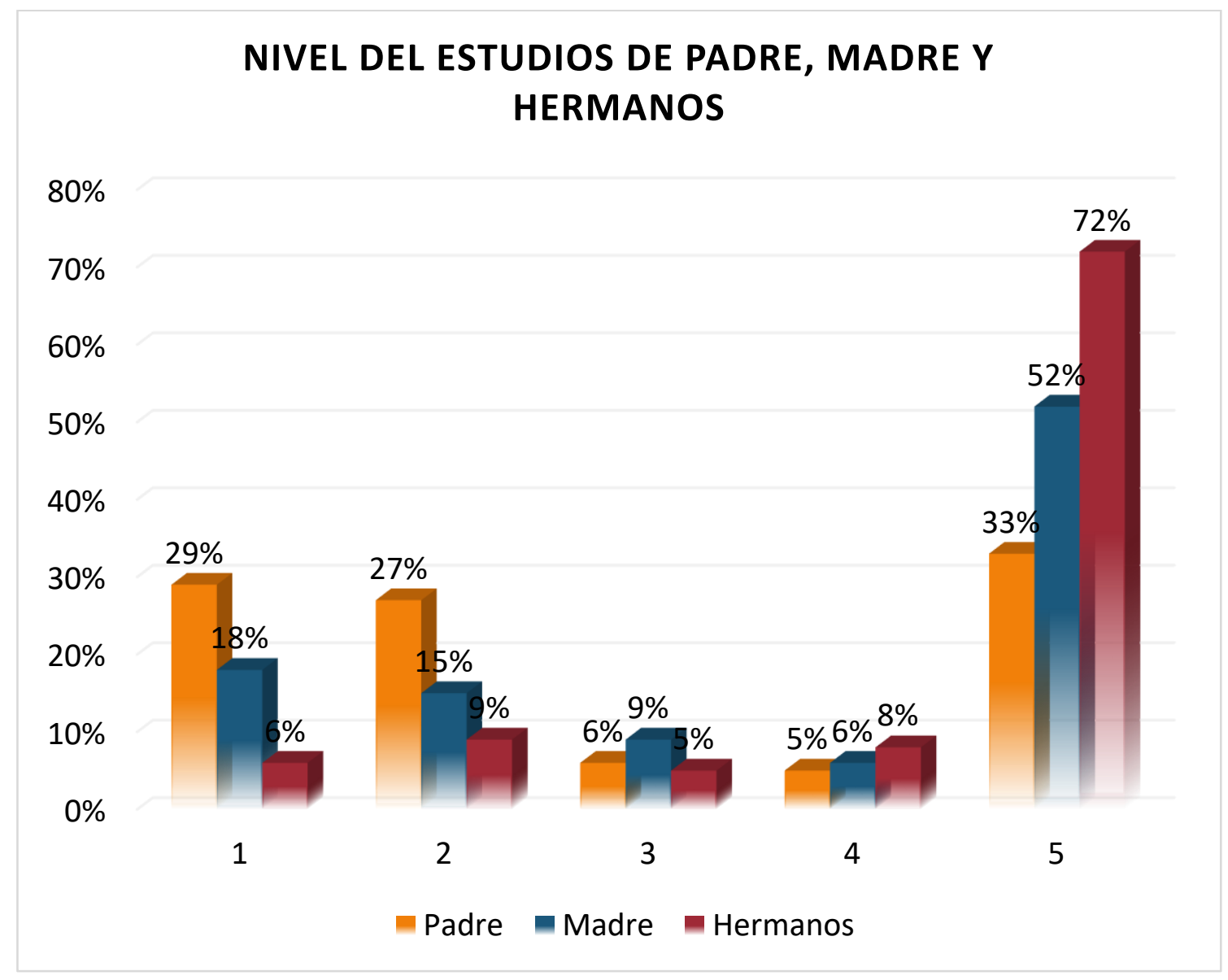

Fuente: Elaboración propia.

(Lehmann, 2007) señaló que los EPG abandonan un programa de estudio antes que otros alumnos, aun cuando poseen un alto rendimiento académico. Por su parte, (Chen, 2005) mostró que el $43 \%$ de los EPG que se matricularon en una institución postsecundaria no se graduó; en cambio, el 68\% de los estudiantes que tenía algún padre con un título profesional completó el programa de estudios.

El Índice de Progreso Social es la única herramienta de medición que se enfoca de manera integral y sistemática exclusivamente en las dimensiones no económicas del desempeño social en todo el mundo con datos transparentes y procesables.

El progreso en las cuestiones sociales no acompaña automáticamente al desarrollo económico. El aumento de los ingresos generalmente trae consigo importantes mejoras en áreas como el acceso al agua potable, el saneamiento, la alfabetización y la 
educación básica. Pero, en promedio, la seguridad personal no es mejor en los países de ingresos medios que en los de ingresos bajos y, a menudo, es peor. En general, el Índice de Progreso Social proporciona el primer marco concreto para realizar evaluaciones comparativas y priorizar una agenda de acción que promueva el desempeño tanto social como económico. (Green, et. al, 2020)

El progreso social se ha convertido en una agenda cada vez más crítica para los líderes de gobierno, las empresas y la sociedad civil, la medición de este índice da parámetros para comparar el nivel de vida de los egresados antes y después de sus estudios universitarios, sin relacionarlos exclusivamente al aspecto económico, si no más bien a un aspecto social de influencia en la economía de los mismos.

Partiendo de esta premisa se adaptaron los indicadores estándares para la evaluación del factor socioeconómico cambiante en las vidas de los egresados Fig $\mathrm{N}^{\circ} 2$.

Figura $\mathbf{N}^{\circ}$ 2: Índice nacional de progreso social.

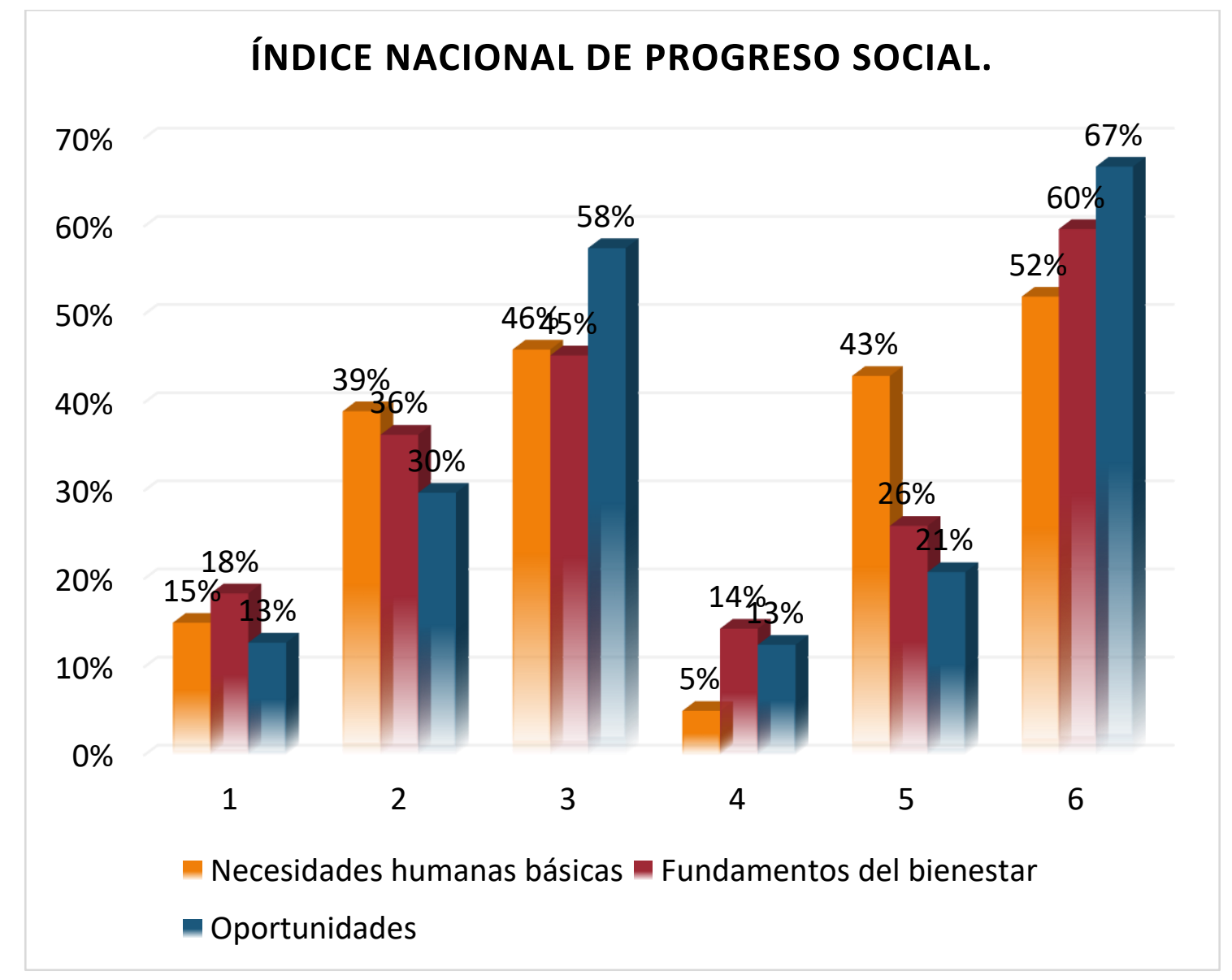

Fuente: Secretaría técnica de planificación de desarrollo económico y social (2018). 
Los resultados presentados en la Fig. $\mathrm{N}^{\circ} 2$ indican una mejora importante del índice de progreso social en los egresados, especialmente en el fundamento de bienestar donde los participantes afirman que luego del egreso mejoraron sus canciones de acceso a telefonías, internet, tv, recolección de basuras y disminuyeron la deforestación a causas de prácticas más sustentables en sus actividades. Del mismo modo, posterior al egreso se evidencian mejor cobertura de las necesidades básicas en sus hogares como las oportunidades de progreso.

Existen diferencias notorias entre los EPG con el resto de los estudiantes, son características demográficas y sociales las que hacen las diferencias, la autopercepción sobre su rendimiento académico, el apoyo familiar, entre otros, contrastes hallados por (Ishitani, 2003). De estudios antecedentes a este, se evidencia que los EPG tienden a pertenecer más al sexo femenino, son por los general son integrantes de un grupo racial/étnico minoritario, poseen un ingreso económico familiar inferior al promedio y tienen aspiraciones educativas muy bajas (Mehta et al., 2011).

\section{CONCLUSIONES}

Mundialmente y en términos de educación superior, se atraviesa una época de expansión, sin precedentes, ésta va acompañada de cambios políticos y socioeconómicos según (Teixeira, Landoni, 2017). La demanda de educación superior aumenta y esto se relaciona estrechamente al bienestar de las personas, a la posibilidad que estas tienen de conseguir empleo a través de su formación y obtención de títulos de grado que la certifiquen. Del mismo modo, (Zhen, Dominguez, 2017) afirman que el desarrollo científico y tecnológico, así como las políticas de reducción del financiamiento de las instituciones públicas, entre otros han constituido en el incentivo del aumento de las instituciones de educación superior privadas.

La hipótesis, planteada al inicio de la investigación, es comprobada a través de los resultados obtenidos.: Los Estudiantes de primera generación (EPG), graduados en la Facultad de Ciencias Contables, Administrativas y Económicas de la Universidad Nacional de Pilar, han obtenido un impacto socio-económico positivo en sus familias.

\section{A modo de recomendación se siguiere cuanto sigue:}

- Generar capacitaciones y orientaciones básicas que permitan a los docentes comprender y generar acciones pedagógicas que consideren las características de un EPG. 
- Impulsar una cultura inclusiva en las instituciones cuyo foco sean todos los estudiantes que la componen y que se encuentre sostenida por una política inclusiva que se sustente desde sus bases

\section{REFERENCIAS BIBLIOGRÁFICAS}

Allen, J. (2003). La medición de las competencias de los titulados universitarios. Universidad de León.

Araneda, C.; Gairín, J., Pedraja, L., Rodríguez, E. (2018). Percepciones sobre el perfil del estudiante universitario en el contexto de la educación superior de masas: aproximaciones desde Chile. Interciencia, vol. 43, núm. 12, 2018, diciembre, pp. 864-870 Asociación Interciencia Venezuela.

Arocena, J. (1995). El desarrollo local: un desafío contemporáneo, Caracas, Editorial Nueva Sociedad.

Bourdieu, P. (1997). Capital Cultural, Escuela y Espacio Social, Siglo XXI editores. Primera edición en español, 1997 Segunda edición en español, 1998 @ siglo xxi editores, s. a de c. v. isbn 968-23-2054-2

Brunner, J. (2015). Medio siglo de transformaciones de la educación superior chilena: Un estado del arte. En Bernasconi A (Ed.) La Educación Superior de Chile. Transformación, Desarrollo y Crisis. Universidad Católica. Santiago, Chile. pp. 21-108.

Carnoy, M., (2017). Global: Does Higher Education Expansion Equalize Income Distribution. In Understanding Global Higher Education, 13-15.

Charlot, B. (1999). La relación con el conocimiento en un entorno popular. Investigación en escuelas secundarias suburbanas. Paris: Anthropos.

Choy, S. (2001). Students whose parents did not go to college: Postsecondary access, persistence, and attainment. National Center for Education Statistics, Washington.

Congreso Nacional del Paraguay. (15 de 01 de 2021). Ley $\mathrm{N}^{\circ}$ 6628/2020. Ley de Arencel Cero para las universidades públicas del Paraguay. Asunción, Paraguay: Congreso Nacional de la Rca. del Paraguay.

Courtois, A. (2018). 'It doesn't really matter which university you attend or which subject you study while abroad.' the massification of student mobility 
programmes and its implications for equality in higher education. Eur. J. Higher Educ. 8: 1-16.

Dirección General de Estadísticas, Encuestas y Censos (DGEEC), (2002). Censo Nacional de Población y Vivienda.

Espinoza, O., González, L. (2017). Access of disadvantaged students to higher education in Chile: Current scenarios and challenges. En Shah M, Whiteford G (Eds.) Bridges, Pathways and Transitions. Chandos. Oxford, UK. pp. 103-126.

Flanagan, A. (2016). Extending the literature on first-generation university students: A phenomenological study of Chilean experiences [tesis inédita de posgrado]. University of Nebraska, Lincoln.

Flanagan, A. (2017). Experiencias de estudiantes de primera generación en universidades chilenas: realidades y desafíos. ANUIES. Revista de la Educación Superior, 46(183), 87-104. Obtenido de http://creativecommons.org/licenses/by-nc-nd/4.0/

Forrest, E., Bennett, C., (2018). First-Generation Students. National Center for Education Statistics. Recuperado el 19 de 09 de 2021, de https://nces.ed.gov/pubs2018/2018421.pdf

Gofen, A. (2009). Capital familiar: Cómo los estudiantes de educación superior de primera generación rompen el ciclo intergeneracional. Relaciones familiares, 58, 104-120.

Green, M., Harmacek, J., Krylova, P. (2020). Índice de progreso social 2020.Resumen Ejecutivo. socialprogress.org

Hernández, R., Fernández, C., Baptista, P. (2014). Metodología de la Investigación. Roberto Hernández Sampieri. . (6ta ed.). México, México DF.: McGrawHill/Interamericana Editores, SA.

Ishitani, T. (2003). A longitudinal approach to assessing attrition behavior among firstgeneration students: Time-Varying effects of pre-college characteristic. Research in Higher Education, 48 (4) (2003), pp. 403-434

Lahire, B. (1995). Problemas escolares y miseria en los círculos populares.. Paris: Le Seuil. Las Universidades: Una aproximación al contexto, estructura y resultados. 
Lehmann, W. (2007). I just didn't feel like I fit in: The role of habitus in university drop-out decisions. Canadian Journal of Higher Education, 37 (2) (2007), pp. 89-110.

Linne, J, (2018). El Deseo de Ser Primera Generación Universitaria. Ingreso y Graduación en Jóvenes de Sectores Populares.

López, F. (2007). Educación Superior Internacional Comparada, Escenarios, Temas y Problemas.

Marginson, S., (2017). Elite, mass, and high-participation higher education. En Shin J, Teixeira P (Eds) Encyclopedia of International Higher Education Systems and Institutions. Springer. Dordrecht, Holanda. pp. 1-9.

Mehta, S., Newbold, J., O’Rourke, M. (2011). Why do first-generation students fail? College Student Journal, 45 (1) (2011), pp. 20-35

O'Shea, S. (2016). Avoiding the manufacture of 'sameness': first-in-family students, cultural capital and the higher education environment. Higher Educ. 72: 59-78.

Sabino, C. (1996). El Proceso de Investigación. Buenos Aires. Ed. Lumen - Humanitas. 1996

Sémbler, C. (2006). Estratificación social y clases sociales. Una revisión analítica de los sectores medios. Serie Políticas Sociales Nº125, CEPAL

Soto, V., (2015). Estudiantes de primera generación en Chile: una aproximación cualitativa a la experiencia universitaria, Universidad de Concepción (Chile)

Stock, M. (2018). The transition from 'Rank Equality' to Vertical Differentiation in the German higher education sector. En Bloch R, Mitterle A, Paradeise C, Peter T (Eds.) Universities and the Production of Elites. Macmillan. pp. 149-171.

Tajfel, H. (1981). Human groups and social categories. Cambridge University Press (Versión en castellano, Barcelona: Herder, 1984).

Teixeira, P., Landoni, P. (2017). The rise of private higher education. En Rethinking the Public-Private Mix in Higher Education, 21-34. Sense.

Terenzini, P., Leonard, P. Yaeger, M., Ernest, T. Pascarella, A. (1996). First-generation college students: Characteristics, experiences, and cognitive development. Research in Higher Education, 37 (1996), pp. 1-22

Trow, M., (1973). Problems in the Transition from Elite to Mass Higher Education. Carnegie Commission on Higher Education. Berkeley, CA, EEUU. 57 pp. 
Viceministerio de Educación Superior (VES), (2013). Informe del estudio basado en las encuestas realizadas por el Viceministerio de Educación Superior MEC, CIIE.

Zhen, Y., Domínguez, J., (2017) La educación superior privada en el modelo de socialismo con características chinas/Private Higher Education in Socialism with Chinese Characteristics. Rev. Cub. Educ. Sup. 36: 137-149. 